\title{
Session Table Architecture for Defending SYN Flood Attack
}

\author{
Xin Li, Zhenzhou Ji, and Mingzeng Hu \\ School of Computer Science and Technology, \\ Harbin Institute of Technology, Harbin, China \\ \{lixin, jzz, mzhu\}@pact518.hit.edu.cn
}

\begin{abstract}
Stateful Inspection has become a classical technology for network firewall. Existing session table architectures of Stateful Inspection firewalls cause high time cost of timeout processing. A new architecture is proposed. The new architecture divides a session entry into two separate parts, and designs different data structures for each other. On the base of multi-queue architecture, dynamical timeouts according to available resource improve securities of protected hosts against SYN flood attack. Experimental results show that the new architecture can work well in Gigabit Ethernet network.
\end{abstract}

\section{Introduction}

Stateful Inspection refers to an extension of packet-by-packet filtering process that tracks individual flows, enabling policy checks that extend across series of packets [1]. Many firewalls have implemented Stateful Inspection technology, such as Cisico PIX [2], 3COM Secure Gateway [3], Netsreen Firewall [1] and Checkpoint FW-1 [4]. Stateful Inspection requires a session table whose entries typically record source and destination IP addresses and port numbers. For each packet, the session table is looked up for a match. A session entry in the format <src-addr, src-port, dst-addr, dstport, ip-p, state, time $>$ is created when the first packet appears from a flow previously not tracked. Subsequent packets of an established session are checked against the session table rather than against the Rule Base. The performance of Stateful Inspection firewall mainly depends on the performance of processing session table.

Under normal operating condition, all session table entries represent a valid flow. However, abnormal events can create an excessive number of invalid entries in the table. A representative example is the DoS attack using TCP SYN packets. Suppose TCP SYN packets from typically spoofed source addresses go out through a Stateful Inspection firewall in avalanche, triggering the creation of corresponding session entries in the table. When the session table is inundated with invalid entries, performance and security become concerns. So timeout processing is essential for TCP to purge the inactive session [5]. Firewalls are better suited to fight the attack because they tend to be designed to examine packets and maintain connection and state information of session traffic [6].

The TCP connection setup delay is defined to be the time elapsed between the transmission of the first SYN packet and the reception of the corresponding ACK. 
The connection setup delay is usually much less than 1 second. At 1 second, more than $93 \%$ of the connections are established [5]. Furthermore, after a connection is established, the timeout is automatically set to 3600 seconds, which may cause session table quickly filled up. So the default timeout of some firewall is too long, which cause the host easy to be crashed. So, shortening TCP idle timeout will decrease the opportunity to fill session table [5]. But if timeouts are too short, session table will be inserted and deleted frequently.

Processing session timeout is essential for connection-level monitoring devices such as Stateful Inspection firewalls in order to minimize security holes. Adequate session timeouts can improve the security of both protected host and firewall itself.

This paper proposed a new architecture for session processing, which improves the timeout processing performance of session table effectively, and securities of both protected hosts and firewall itself.

\section{Session Table Processing}

Generally, <src-addr, src-port, dst-addr, dst-port, ip-p > is used to identify a unique session, which is called SID in this paper. For each arriving packets, session table is looked up for a match. If packets belong to an existing session, session state and session time will be updated. If a session entry has overtime, it will be deleted.

Generally, there are 4 kinds of session table operation: match session table with packets' sessionID, update an entry's time and state, insert a new entry and delete an overtime entry, which are shown in Fig.1. Because an update always is after a match, we call them a match-and-update operation.

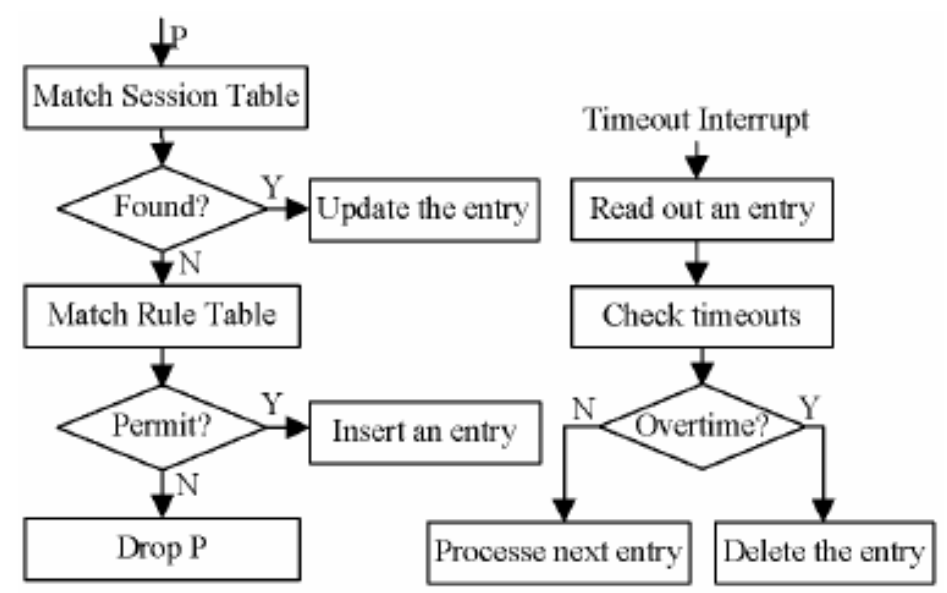

Fig. 1. Session table operations

Required time for inserting an entry $\left(T_{i n s}\right)$, deleting an entry $\left(T_{d e l}\right)$, and match-andupdate $\left(T_{\text {mau }}\right)$ are three key parameters to the performance of Stateful Inspection firewalls. Because memory access is most time-consuming, we use the required clock cycles of memory accesses to measure $T_{i n s}, T_{d e l}$ and $T_{\text {mau }}$. 


\subsection{Existent Processing Methods of Session Table}

Now all existed firewalls put both SID and <state, time> in a single entry, as Fig.2. Because the number of entries may up to 1 million, and each entry is wider than 128 bits, over $128 \mathrm{Mb}$ memory space is required for session table. Generally, DDR SDRAM is used to store session table.

\begin{tabular}{|l|l|l|l|l|l|l|}
\hline Sre_addr & dst_addr & Src_port & dst_port & protocol & State & Time \\
\hline
\end{tabular}

Fig. 2. General format of session table entry

Because SID is about 128 bits long, comparing a packet SID with session table entries requires an efficient searching algorithm that performs searching without comparing the whole 128 SID bits. One such efficient searching algorithm is PATRICIA. PATRICIA is an algorithm that needn't compare the whole key. PATRICIA is very useful for extremely long key. PATRICIA trie compress all nodes which have oneway branch, it is the lowest trie [7]. But traversing PATRICIA trie to process timeout is time-consuming. In order to improve traversing performance, leaves should be linked to a rope. Fig.3 illustrates the data structure in [8] for fixed-length match, and Fig.4 illustrates the Leaf Rope of PATRICIA trie which is a single linked list. All leaves in the PATRICIA trie are linked as a rope in inserting time order.

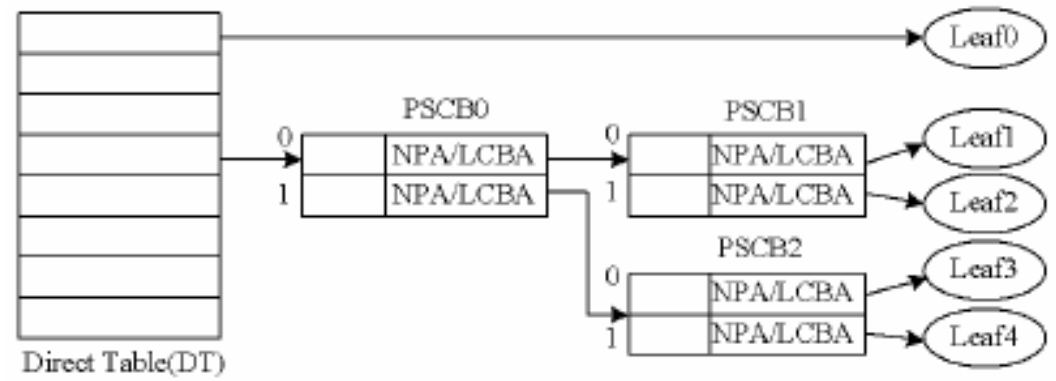

Fig. 3. Data Structure of IBM NP4GS3's FM [8]

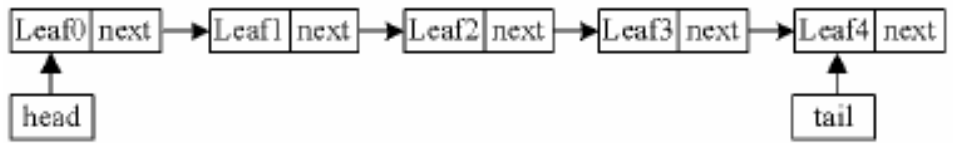

Fig. 4. Leaf Rope structure

$T_{\text {search }}, T_{\text {insert }}$ and $T_{\text {delete }}$ are respectively required time of searching, inserting and deleting an entry in PATRICIA trie. From the processing in Fig.1 and PATRICIA algorithm in [9], we get the following functions, which have considered the time cost 
of applying and reclaiming memory block. The $R_{o t}$ is the ratio of the number of overtime entries to all entries, and $T_{D D R}$ is required clock cycles of accessing DDR memory.

$$
\begin{aligned}
& T_{\text {mau }(1)}=T_{\text {search }}+T_{D D R} \\
& T_{\text {ins }(1)}=T_{\text {insert }}+2 T_{D D R} \\
& T_{\text {del }(1)}=T_{\text {delete }}+T_{D D R} / R_{\text {ot }}
\end{aligned}
$$

$R_{o t}$ generally is very small, which is far less than 1 , so timeout processing is timeconsuming. When processing many overtime entries once, the time cost of timeout processing is intolerable.

\subsection{Doubly Linked List Structure}

From the timeouts processing of Fig.1, we know that to improve timeout processing performance, the rope should be linked in updating time order. If the rope is linked as a doubly linked list in time order, we need not read many entries when processing timeout. When an entry does not timeout, all subsequent entries do not timeout too. Fig. 5 shows the data structure.

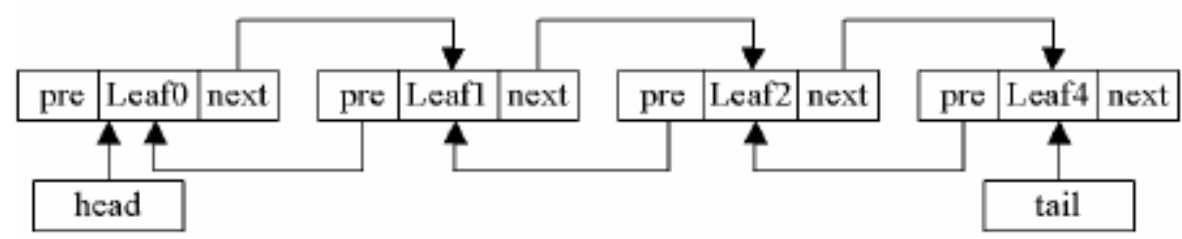

Fig. 5. PATRICIA based doubly linked list

$$
\begin{aligned}
& T_{\text {mau }(2)}=T_{\text {search }}+7 T_{D D R} \\
& T_{\text {ins }(2)}=T_{\text {insert }}+2 T_{D D R} \\
& T_{\text {del(2) }}=T_{\text {delete }}+T_{D D R}
\end{aligned}
$$

This architecture can improve the performance of timeout processing effectively, but it causes the performance of match-and-update down heavily. The main reason is that it needs too many memory accesses when updating session table. It needs 7 memory accesses at least, and DDR is very slow.

\subsection{Proposed Architecture}

Higher speed memory than DDR can improve the performance of session table. In this paper, we proposed a new architecture for session table. We use two kinds of memory to store session table, which can improve the performance of session table greatly. Because SID is wide and session table generally have too many entries, we uses DDR SDRAM to store SIDs PATRICIA trie.

In order to decrease $T_{\text {del( }(1)}$, we use a doubly linked list to organize <state, time> . Fig.6 shows its data structure. ZBT SRAM is used to store the doubly linked list. We use DS1_addr and DS2_addr to relate the two data structures, as Fig.7. 


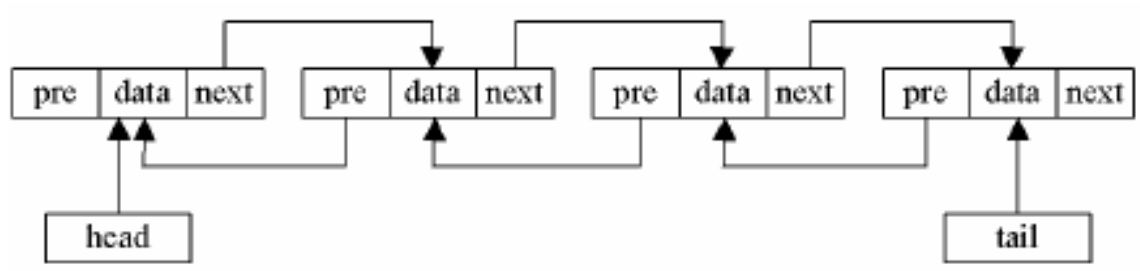

Fig. 6. Doubly Linked Lists for DS2

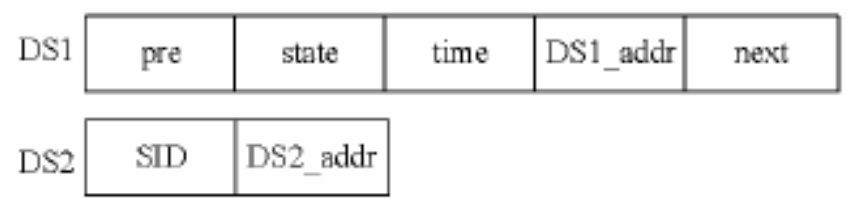

Fig. 7. Entries of DS1 and DS2

Algorithm 1. Insert a session entry.

(1) apply a DDR memory block for DS1 and a ZBT memory block for DS2.

(2) fill the DS1 block with sessionID and the DS2 block's address, and insert it in PATRICIA trie.

(3) fill the DS2 block with state, time and the DS1 block's address, insert it in doubly linked list.

Algorithm 2. Match and update a session entry.

(1) search PATRICIA trie for a match, if matched use DS2_addr as address to read DS2 node.

(2) update the DS2 node with new state and current time.

(3) delete the DS2 node from its old position.

(4) insert the DS2 node in the tail of list, update list's tail.

Algorithm 3. Timeout processing.

(1) when received a timer interrupt, read out the first DS2 node from its head.

(2) compare the time in DS2 with current time to determine whether it is overtime or not.

i (3) if overtime delete the DS2 node, update list's head, and delete the correspondng DS1 node.

(4) process its next node.

Following functions measure the performance parameters of the proposed architecture.

$$
\begin{aligned}
& T_{\text {mau }(3)}=T_{\text {search }}+7 T_{Z B T} \\
& T_{\text {ins }(3)}=T_{\text {insert }}+3 T_{Z B T} \\
& T_{\text {del (3) }}=T_{\text {delete }}+2 T_{Z B T}
\end{aligned}
$$




\subsection{Pipeline the Proposed Architecture}

$T_{\text {search }}, T_{\text {insert }}$ and $T_{\text {delete }}$ only involve DDR SDRAM. Because $T_{D D R}$ needs about 16 cycles for 32 bits memory data bus and 256 bits data, $T_{\text {search }}, T_{\text {insert }}$ and $T_{\text {delete }}$ at least need 32 cycles. $T_{Z B T}$ only involves ZBT SRAM, and $T_{Z B T}$ only need 2 cycles for 36 bits memory data bus and 72 bits data. We get $7 T_{Z B T}<T_{\text {search }}, 3 T_{Z B T}<T_{\text {insert }}$ and $2 T_{Z B T}<T_{\text {delete }}$. If we pipeline DS1 operation and DS2 operation, the performance of session table will only be determined by $T_{\text {search }}, T_{\text {insert }}$ and $T_{\text {delete }}$. Following functions show the performance parameters of the pipelined architecture.

$$
\begin{aligned}
& T_{\text {mau(4) }}=T_{\text {search }} \\
& T_{\text {ins(4) }}=T_{\text {insert }} \\
& T_{\text {del(4) }}=T_{\text {delete }}
\end{aligned}
$$

\subsection{Performance Analysis}

In our design, $T_{D D R}$ equals 16 cycles and $T_{Z B T}$ equals to 2 cycles. From above analyses, we know that $T_{\operatorname{del}(4)}$ is much less than $T_{\operatorname{del}(1)}$, and $T_{\operatorname{mau}(4)}$ is much less than $T_{\operatorname{mau}(2)}$. We can know that the pipelined architecture is the best architecture.

From above analyses, we know that $T_{\text {search }}, T_{\text {insert }}$ and $T_{\text {delete }}$ determine session table's performance. So to minimize $T_{\text {search }}, T_{\text {insert }}$ and $T_{\text {delete }}$ is the most important thing to improve the performance of session table processing. On the base of traditional PATRICIA trie we proposed a new PATRICIA trie, which improves the performance of traditional PATRICIA insertion [9].

\section{Dynamical Timeouts}

\subsection{Queue Structure}

An important advantage of the new architecture is that we can easily organize multiple double linked lists in ZBT SRAM, and each double linked list is called a queue in this paper. We can set dynamical timeouts for each queue, and each queue's timeouts can be different, which can improve securities of both protected hosts and firewall itself.

To illustrate our methods for dynamical timeouts, we use typical topology of network as example, as Fig.8. In this paper, we assume the HTTP serve and FTP serve need be protected against SYN Flood attack, and firewall itself need defense other TCP attack.

We use ZBT SRAM to store DS2 data structure. We design four queues for different functions. Queue 1 and Queue 2 are respectively used to store session tables which have received the SYN/ACK from the HTTP server and FTP server. Queue 3 is used to store session tables which have established TCP connection but not receive any FIN or RST packets. Queue 4 is used to store all others' session tables. Fig.9 shows queues and sessions flow examples. 


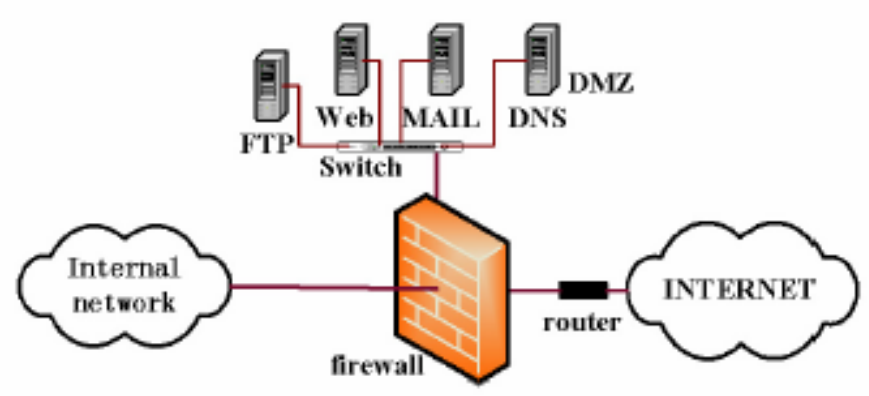

Fig. 8. Typical firewall topology

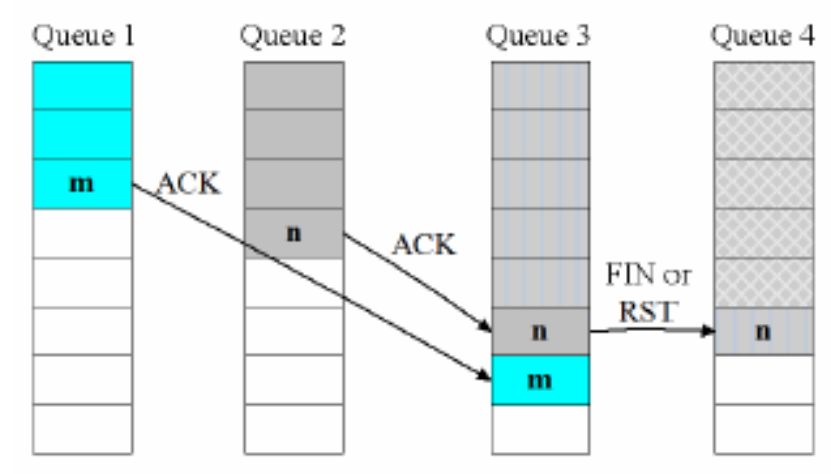

Fig. 9. Four queues used for storing sessions

\subsection{Algorithms for Queues}

For each arriving packet, the PATRICIA Trie is looked up for a match.

Algorithm 6: SYN packets processing.

(1) if find a session entry, drop the packet, else lookup in the rule base for a match.

(2) if does not be allowed, drop it, else use Algorithm 1 to insert the new entry in Queue 1.

Algorithm 7: SYN/ACK packets processing.

(1) if not find a session or the session's state is wrong, drop the packet.

(2) else if destination IP is HTTP server, move the session table entry from Queue 4 to Queue 1, else if destination IP is FTP server, move the session table entry from Queue 4 to Queue 2, else move the session table entry to the tail of Queue 4.

Algorithm 8: ACK packets processing.

(1) if not find a session or the session's state is wrong, drop the packet.

(2) else move the session table entry to the tail of Queue 3.

Algorithm 9: packets with FIN or RST.

(1) if not finding a session or the session's state is wrong, drop the packet.

(2) else move the session to the tail of Queue 4. 


\subsection{Dynamical Timeouts}

For the above four queues, the first two queues is mainly related with the security of protected host, and the last two queue is mainly related with the security of firewall itself. If defining $T$ is the maximum of timeout, $N$ is the whole resource, and $M$ is available resource, we proposed a new timeout model. Fig.10 illustrates timeouts curve with $M$ varying.

$$
\text { Timeout }=T \frac{1}{2^{\left\lfloor\lg \frac{N}{M}\right\rfloor}}
$$

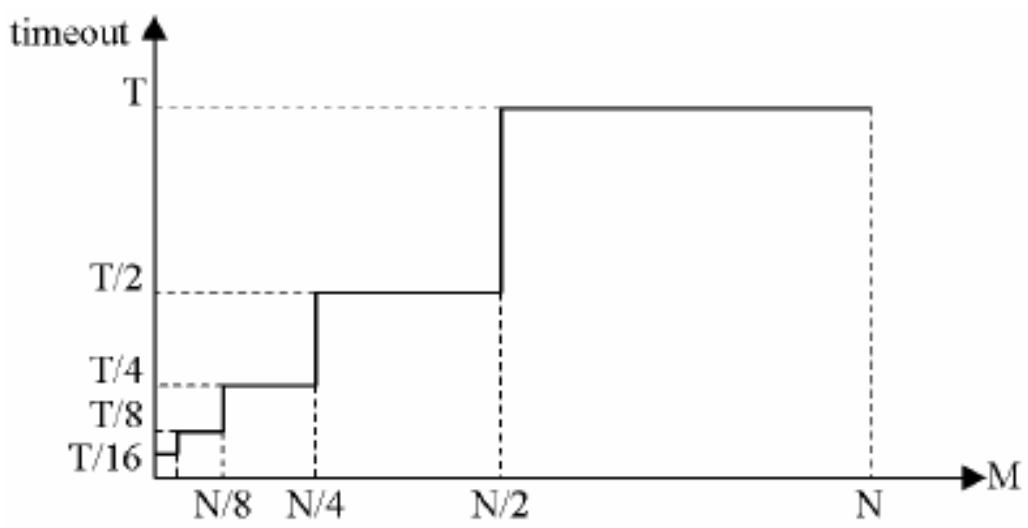

Fig. 10. Timeouts curve

For timeouts of the first two queues, selecting $T$ is 32 seconds, $N$ is configured from user which typically is the number of half open host supported, and $M$ is available resource in protected host. To insure the slow network user can through firewall normally, minimal timeouts of Queue 1 and Queue 2 are 1 second. The $N$ and $T$ may different for the two queues according to users' configuration.

For timeouts of Queue 3, selecting $T$ is 4096 seconds, $N$ equal to the whole space of ZBT memory, and $M$ is available resource in ZBT SRAM. The minimum of timeout is 256 seconds.

For timeouts of Queue 4, selecting $T$ is 32 seconds, $N$ equal to the whole space of ZBT memory, and $M$ is available resource in ZBT SRAM. To insure the slow network user can through firewall normally, its minimum of timeout is 1 second.

When timeouts of any queue has decreased to its minimum, each new connection will purge an oldest connection in the session table.

\subsection{Performances and Securities Analyses}

Dynamical timeouts improve securities of both protected host and firewall itself.

(1) when processing timeout as Algorithm 3, only access part of table entries because the linked list is in the order of updating time, which improves the performance 
of timeout processing. By pipelining, the time cost of timeout processing is equal to the time cost of deleting the DS1 entry.

(2) when updating session table entry as Algorithm 2, By pipelining, the time cost of updating session is equal to the time cost of matching a DS1 entry.

(3) as Algorithm 2, 6 and 7, firewall uses special queues for protected hosts to defense SYN flood, and sets timeouts of Queue 1 and Queue 2 according to available resource of the protected host, which can improve protected hosts' security against SYN Flood. Furthermore, when timeouts of Queue 1 and Queue 2 down to the minimum, each new inserted entry will cause the oldest entry purged, which can insure the protected hosts not crashed.

(4) as Algorithm 2, 7, 8 and 9, firewall sets timeouts of Queue 3 and Queue 4 according to available resource of firewall itself, which can improve both protected hosts and firewalls' security. Furthermore, when timeouts of Queue 3 or Queue 4 down to the minimum, each new inserted entry will cause the oldest entry purged, which can insure the firewall not crashed.

\section{Experimental Design and Performance Analyses}

For high speed network, ASIC is often used to improve performance of network device. We implement an ASIC for the proposed session table architecture and proposed algorithms. We use two methods to reduce the depth of PAT-FM trie. One is to hash SID, the other is to make use of 4-ary PATRICIA trie. We use a Xilinx FPGA(XC2V3000) to implement the whole Stateful Inspection firewall which supports 3 Gigabit Ethernet ports and a PCI interface. PATRICIA trie is stored in DDR SDRAM, doubly linked list is stored in ZBT SRAM.

We use random 128 bits data to test the performance of session table insertion, search and deletion. Table 1 shows the experimental results. We can know that performances of session entries insertion, search and deletion are very close. All of these performances are mainly determined by PATRICIA's performance.

Furthermore, we mainly test the performance of lookup session table for a match, because lookup performance is the most important parameter for Stateful Inspection firewalls. Fig.11 shows the experimental result, the ASIC for the new architecture and new algorithms can do 2.78 million lookups even the number of session entries up to 1 million. If all packets are smallest packet (64 bytes), the application-specific hardware can process $1.7 \mathrm{Gbps}$ ' traffic. And when the average size of packets is 128 bytes, the traffic can up to $3.4 \mathrm{Gbps}$. So, we can know that this ASIC can work well in the Gigabit edge network device.

Table 1. Operation numbers per second

\begin{tabular}{ccc}
\hline Search & Insertion & Deletion \\
\hline $2,464,274$ & $2,796,528$ & $2,471,232$ \\
\hline
\end{tabular}




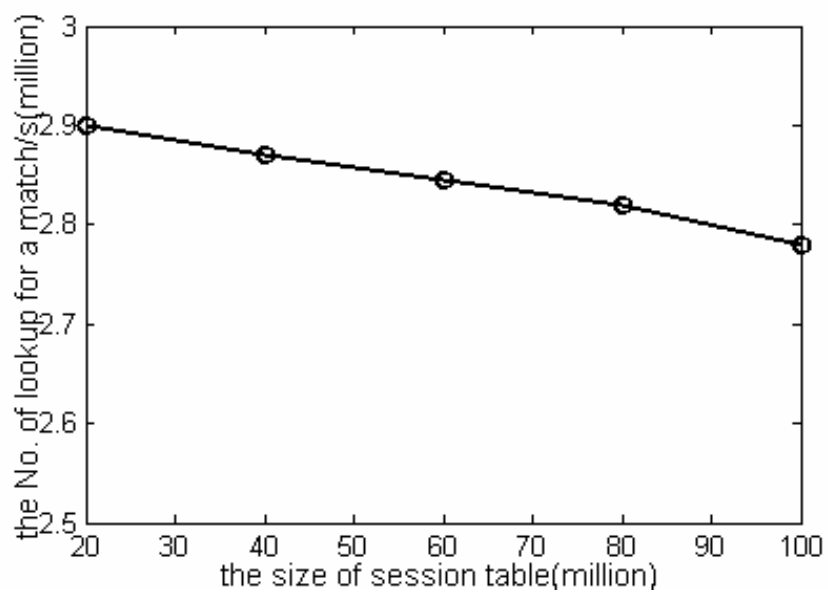

Fig. 11. Lookup performance of session table

\section{Conclusions}

The Stateful Inspection technology is a key to network firewall, and the performance of session table is a key to the Stateful Inspection technology. The new architecture improves performances of both timeout processing and session updating. Dividing session entry into two separate parts and designing different data structures for each other improves the performance of session table. By pipelining operations of the two parts, the performance of session table is only determined by PATRICIA trie's performance. The new fixed-length match PATRICIA algorithm can improve insertion performance effectively.

Because the new architecture sets dynamical timeouts according to available resource of both protected host and firewall, which insure both protected hosts and firewall itself not SYN-Flooded. The specialized hardware for the proposed architecture is implemented in FPGA, which improve firewall's performance further. By analyzing the architecture and experimental results, the new session table architecture can work well in Gigabit Ethernet network.

\section{References}

1. Stateful-inspection firewall: The Netscreen way. http://www.netscreen.com/products/firewall_wpaper.html.

2. David, W., Chapman, Jr., Andy, F.: Cisco Secure PIX Firewalls, Cisco Press (2001)

3. Http://www.3com.com/other/pdfs/products/en_US/ 400742. pdf.

4. Marcus, G., Steven, B.: Check Point Firewall-1 Administration Guide (2001)

5. Inhye, Kang, Hyogon, Kim: Determining embryonic connection timeout in Staeful Inspection, IEEE 2003 International Conference on Communication. Anchorage, USA (2003) $458-462$ 
6. Noureldien, A.N.: Protecting Web Servers from DoS/DDoS Flooding Attacks, International Conference on Web-Management for International Organization. Geneva (2002)

7. Okuno, M., Ando, K., Aoe, J.: An efficient compression method for Patricia tries, IEEE International Conference on Computational Cybernetics and Simulation, Vol.1 (1997) 415420

8. IBM Co.: IBM NP4GS3 DATAsheet (2001)

9. Li, X, Hu, M.Z, Ji, Z.Z, A Hardware-Based PATRICIA Algorithm for Fixed-length Match, Computer Research and Development (2005) 951-957 